\title{
Patch exclusion technique with Amplatzer septal occluder device for the treatment of postinfarction ventricular septal defect
}

\author{
Isidore Dinga Madou, MD, Adam R. Williams, MD, and Jeffrey G. Gaca, MD, Durham, NC
}

$\checkmark$ Video clip is available online.

\section{CASE REPORT}

\section{Patient 1}

A 73-year-old man with a history of hypertension, type 2 diabetes mellitus, and hyperlipidemia presented to our emergency room with a 3-day history of chest pain radiating to the left arm and jaw claudication. Left heart catheterization revealed 3 -vessel disease, including $100 \%$ occlusion of the proximal right coronary artery (RCA). A drug eluting stent was placed to the RCA and ticagrelor was started. A transthoracic echocardiogram showed a large inferior ventricular septal defect (VSD) with left-right flow. An intra-aortic balloon pump (IABP) and a pulmonary artery catheter were placed. He was bridged with cangrelor and taken to the operating room 5 days later.

After median sternotomy, the patient was placed on cardiopulmonary bypass (CPB) via ascending aorta and bicaval cannulation. A vertical ventriculotomy was performed in the infarcted tissue. An inferior VSD was identified (Figure 1, $A$ and $B$ ). Based on the transesophageal echocardiographic measurement and the direct intraoperative measurement of the defect with a ruler and an upsize of $10 \%$ to $20 \%$, a 28-mm Amplatzer septal occlude device (ASOD; Abbot, Chicago, Ill) was directly deployed in the defect by the surgeon (Figure 1, $C$, and Video 1). Interrupted 20 Ethibond (Ethicon, Somerville, NJ) pledgeted sutures

\section{Patient 2}

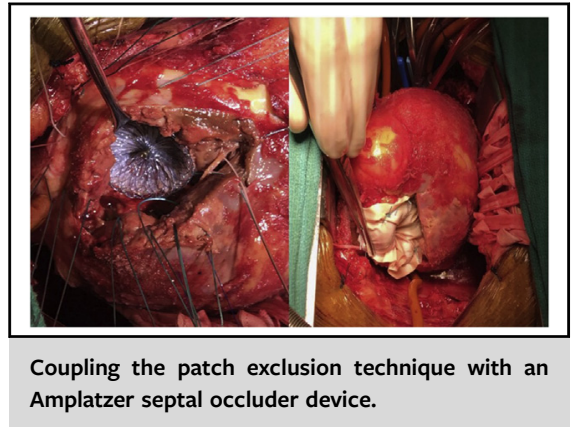

\begin{abstract}
CENTRAL MESSAGE
This synergistic repair confers a greater chance of eliminating the VSD and its associated intracardiac shunting, potentially improving early recovery, hospital discharge, and overall survival.
\end{abstract}

See Commentaries on pages 202 and 204 .

were placed circumferentially around the defect, with each suture passing through the device as well. A patch exclusion repair was performed by passing the sutures through a bovine pericardium patch and tied down (Figure 1,D). The ventriculotomy was closed with reinforced felt strips. ${ }^{1}$ The patient was successfully weaned off $\mathrm{CPB}$ with IABP support and minimal inotropes. The transesophageal echocardiogram after CPB revealed no residual VSD (Figure 2, $A$ and $B$ ). The sternum was left open because of poor right ventricle function, with delayed closure performed 2 days later.

The postoperative course was significant for acute hypoxemic respiratory failure requiring tracheostomy, atrial fibrillation requiring cardioversion, and acute renal failure requiring temporary hemodialysis. The patient had a full recovery of his renal function and was discharged to a rehabilitation facility after 1 month. The 2-month follow-up echocardiogram demonstrated no residual VSD (Figure 2, $C$ and $D$ ).

A 53-year-old man with a history of human immunodeficiency virus, hypertension, active smoking, and alcohol 


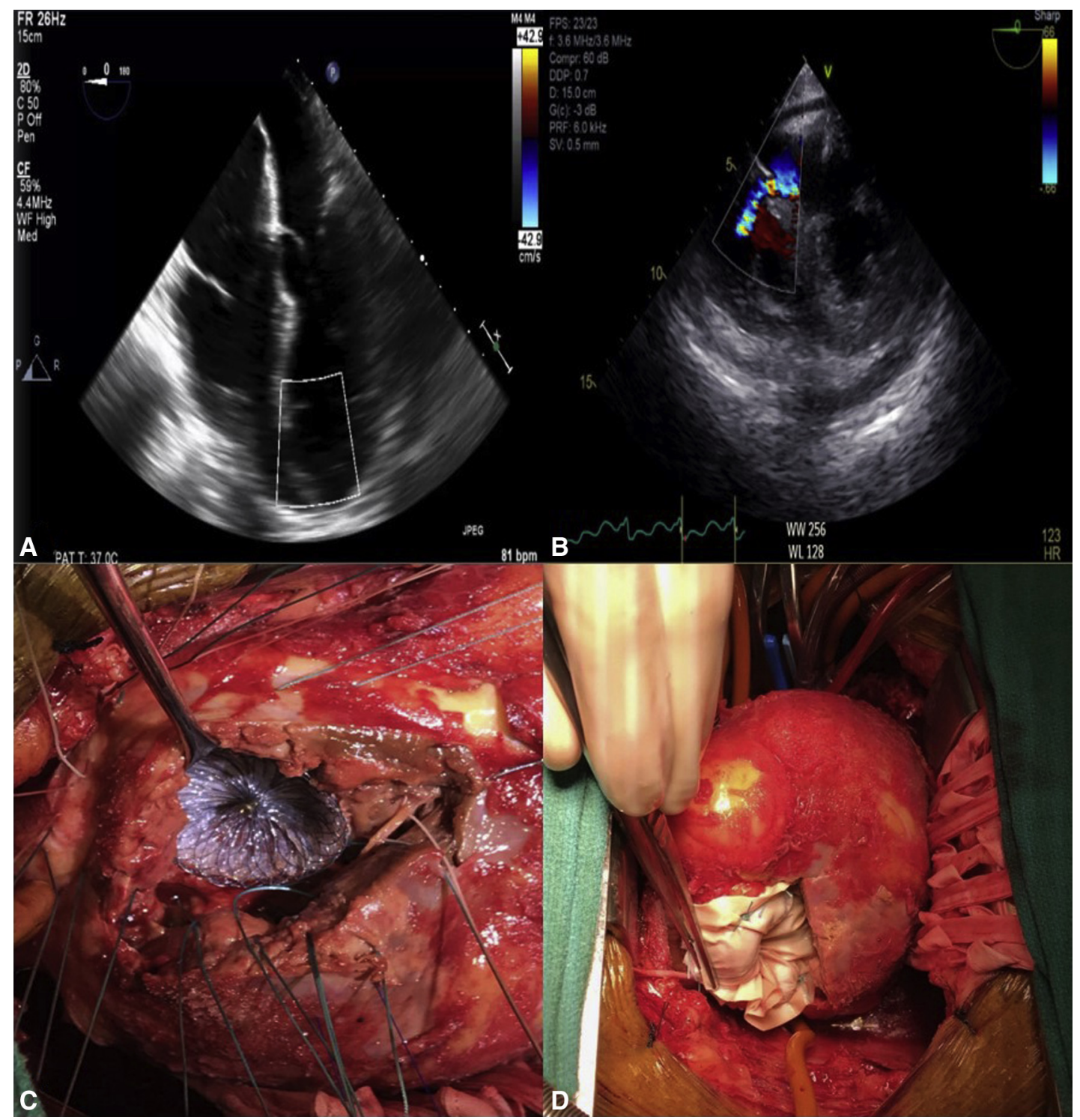

FIGURE 1. Transesophageal echocardiogram, 4-chamber view, showing (A) ventricular septal defect and (B) short axis with color Doppler of left-to-right shunt. C, Intraoperative photograph showing deployment of an Amplatzer septal occluder device through the ventriculotomy under direct visualization. D, The patch exclusion technique with a bovine pericardial patch on top of the Amplatzer septal occluder device in the left ventricle secured with 2-0 Ethibond MH pledgeted sutures going through the septum, occluder device, and then the patch.

abuse had presented with increasing shortness of breath, lethargy, chest pain, and orthopnea. A new loud systolic murmur was noted on physical examination, and a chest radiograph showed pulmonary edema. He was admitted and treated with intravenous diuretics. However, the patient left against medical advice before an echocardiogram had been obtained. He presented to our outpatient clinic 3 days later with progressive shortness of breath, weight gain, and associated chest discomfort. An echocardiogram showed inferior akinesis and a large inferior VSD. Left heart catheterization demonstrated isolated posterior descending artery territory infarct (99\% stenosis) with moderate atrioventricular groove RCA disease and severe mid-left anterior descending disease. He was taken to the operating room 2 days after readmission.

After placement of an IABP and median sternotomy, the left internal mammary artery was harvested, and the patient was placed on CPB via the ascending aorta and bicaval cannulation. Left internal mammary artery to left anterior descending anastomosis was completed. The ventriculotomy revealed a $20-\mathrm{mm}$ inferior VSD, which was repaired as described for patient 1 using a 24-mm ASOD and bovine pericardial patch. The patient was successfully weaned off of CPB with IABP and minimum inotropes. The 


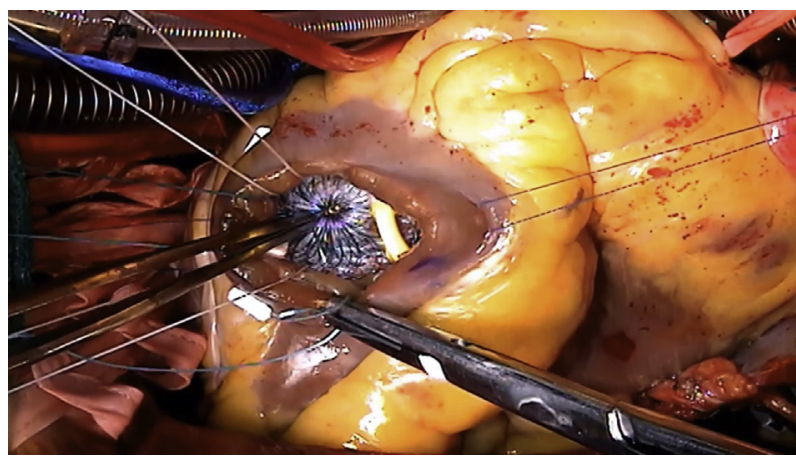

VIDEO 1. This is a video of a third case that we performed after submission of our report. Please note that a vertical ventriculotomy was performed in the right ventricle, the location of the infarcted tissue. A ventricular septal defect was identified. Based on the transesophageal echocardiographic measurement and the direct intraoperative measurement of the defect with a ruler and after an upsize of $10 \%$ to $20 \%$, an Amplatzer septal occluder device (Abbot, Chicago, Ill) was directly deployed in the defect by the surgeon. Interrupted 2-0 Ethibond (Ethicon, Somerville, NJ) pledgeted sutures were placed circumferentially around the defect, with each suture passing through the device as well. A patch exclusion repair was performed by passing the sutures through a bovine pericardium patch and tied down. The ventriculotomy was closed with reinforced felt strips. Video available at: https://www.jtcvs.org/article/S2666-2507(20)30193-0/fulltext. transesophageal echocardiogram after CBP revealed unchanged biventricular function and no residual VSD. The patient was decannulated and the chest closed.

His postoperative course was generally unremarkable, except for a return to the operating room for bleeding on the day of surgery. He was discharged home on postoperative day 5 . He had continued to do well at his postoperative clinic visit. In this case series, because no personal health information that could identify the patients was provided, our research policy does not require informed consent for reporting these cases.

\section{DISCUSSION}

Many techniques are available for postinfarction VSD repair. However, the overall mortality has remained very high. ${ }^{1-3}$ Open surgery with deployment of an ASOD alone has also been reported with variable success. ${ }^{3}$ The percutaneous approach for placement of an ASOD has been essentially limited to treating recurrent and/or residual postinfarction VSD after surgical repair owing to the high rate of failure and mortality as a single treatment modality. ${ }^{4}$

We report a novel technique combining surgical exclusion patch closure with an ASOD. We hypothesized that

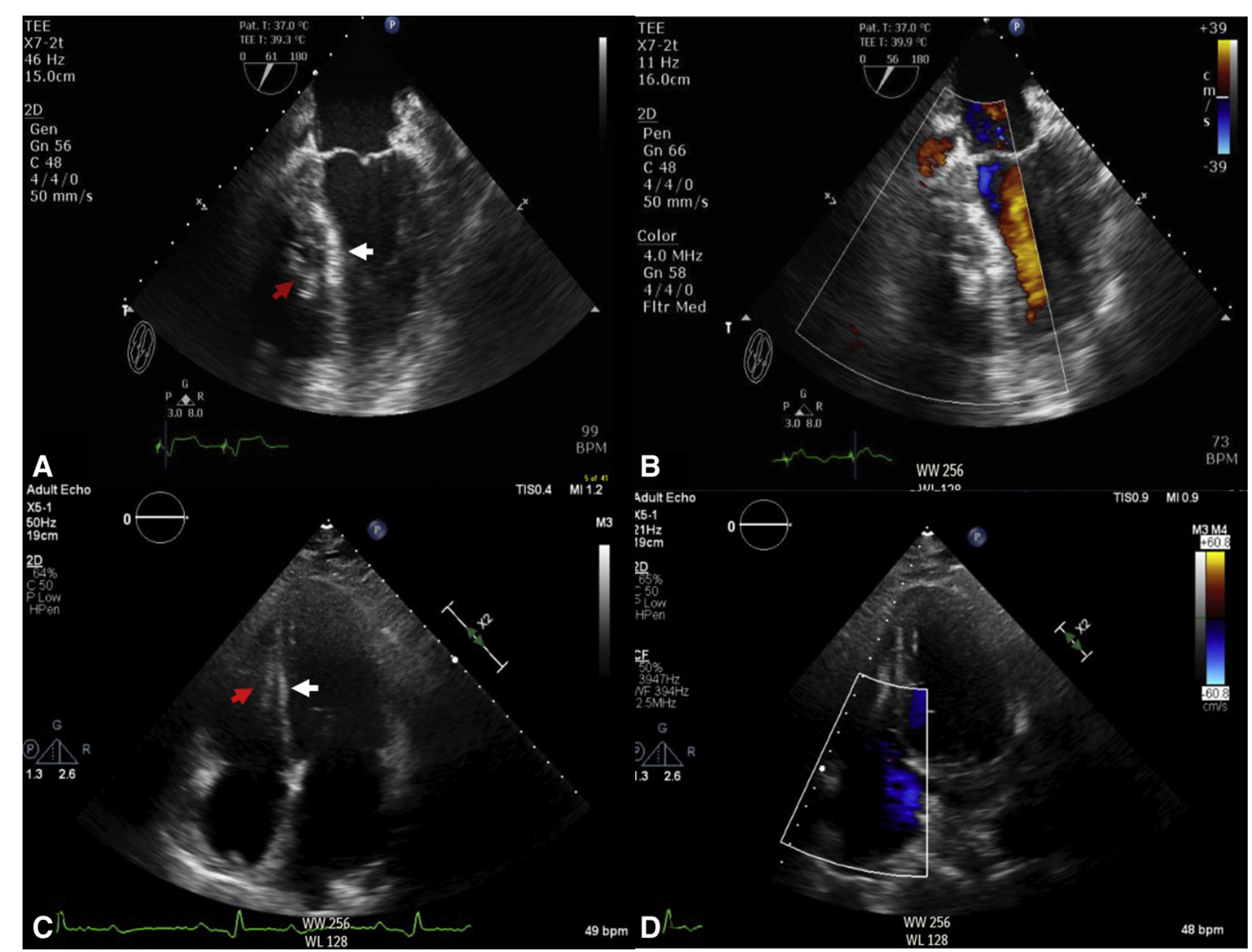

FIGURE 2. Immediate postrepair transesophageal echocardiogram, 4-chamber view, showing (A) ventricular septal defect repair with Amplatzer septal occluder device (red arrow) and patch (white arrows) and (B) color Doppler with no residual left-to-right shunt. Two-month postrepair transthoracic echocardiogram 4-chamber view showing (C) ventricular septal defect repair with Amplatzer occluder device (red arrow) and patch (white arrow) and (D) color Doppler with no residual left-to-right shunt. 
this technique would reduce, not only the rate of ASOD failure because it is deployed under direct vision, but also the rate of patch dehiscence because it is reinforced with the rigid ASOD. This synergistic repair confers a greater chance of eliminating the VSD and its associated intracardiac shunting and negating the potential right-toleft shunt and its associated complications that have often been reported when a left ventricular assist device or an Impella (Abiomed, Danvers, Mass) has been used in these patients. ${ }^{5}$ Thus, use of this synergistic repair will potentially improve early recovery, hospital discharge, and overall survival.

In conclusion, no consensus has been reached for the optimal surgical treatment option for repair of postinfarction VSDs. Coupling the patch exclusion technique with an ASOD for the treatment of postinfarction VSD is a dependable technique with excellent short-term results. However, the long-term outcomes of this approach remain unknown.

\section{References}

1. David TE, Dale L, Sun Z. Postinfarction ventricular septal rupture: repair by endocardial patch with infarct exclusion. J Thorac Cardiovasc Surg. 1995;110:1315.

2. Daggett WM. Postinfarction VSD repair: retrospective thoughts and historical perspective. Ann Thorac Surg. 1990;50:1006.

3. Lee MS, Kozitza R, Mudrick D, Williams M, Lodge AJ, Harrison JK, et al. Intraoperative device closure of postinfarction ventricular septal defects. Ann Thorac Surg. 2010;89:e48-50.

4. Maree A, Jneid H, Palacios I. Percutaneous closure of a postinfarction ventricular septal defect that recurred after surgical repair. Eur Heart J. 2006;27:1626.

5. Kshettry V, Salerno C, Bank A. Risk of left ventricular assist device as a bridge to heart transplant following postinfarction ventricular septal rupture. J Card Surg. 1997;12:93-7. 